\title{
Early onset of Friedreich's ataxia in a compound heterozygote
}

\author{
Mary Claire McGovern, Moira Stewart, Patrick J Morrison, David Webb, Stanley Hawkins
}

Craigavon Area Hospital, 68 Lurgan

Road, Portadown, Co. Armagh BT63 5QQ,

Northern Ireland, UK M C McGovern

Department of Child Health, Institute of Clinical Science, Queen's University of Belfast, Grovesnor Road, Belfast BT12 6BJ, Northern Ireland, UK

M Stewart

School of Clinical Medicine, Queen's University of Belfast S A Hawkins

Department of Medical Genetics, Belfast City Hospital, Belfast BT9 7AB, Northern Ireland, UK P J Morrison

Department of Paediatric Neurology, Royal Belfast Hospital for Sick Children, Belfast BT12 6BE, Northern Ireland, UK D Webb

Correspondence to: Dr McGovern

Accepted 15 February 2000

I

II

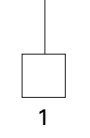

[24]

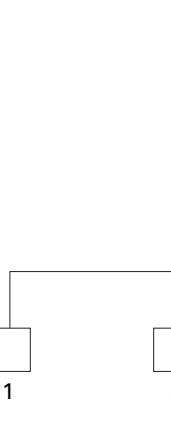

[26]

\begin{abstract}
Friedreich's ataxia (FA) is an autosomal recessive condition caused by a GAA trinucleotide repeat expansion in the $\mathrm{X} 25$ gene on chromosome 9. We describe an unusual form of "pseudodominant" inheritance to illustrate how a diagnosis of FA in a parent does not preclude the diagnosis in the child.

(Arch Dis Child 2000;83:74-75)
\end{abstract}

Keywords: Friedreich's ataxia; genetics; atypical

\section{Case report}

The index case, an 8 year old female Caucasian (see fig 1; III.I) was first seen at the age of 22 months because of unexplained falling and tripping. Pregnancy, birth, and early developmental milestones were normal. She was the only child of non-consanguineous parents. Her father reported normal health although his two brothers had Friedreich's ataxia (FA). At her first visit, growth and general physical examination were normal although reflexes were difficult to obtain. She was kept under review at the child development clinic where examination was normal until the age of 4 when ankle reflexes were absent and intention tremor was noted. She then became increasingly ataxic and by the age of 7 was having difficulty negotiating stairs. Neurological examination at 7 years revealed a mild thoracic scoliosis and bilateral high arched feet. Limb tone was normal and power appeared symmetrical and normal except at the ankle joint where there was mild weakness of dorsiflexion and eversion. Deep

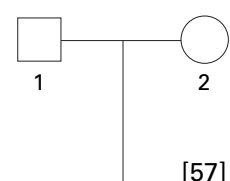

[57]

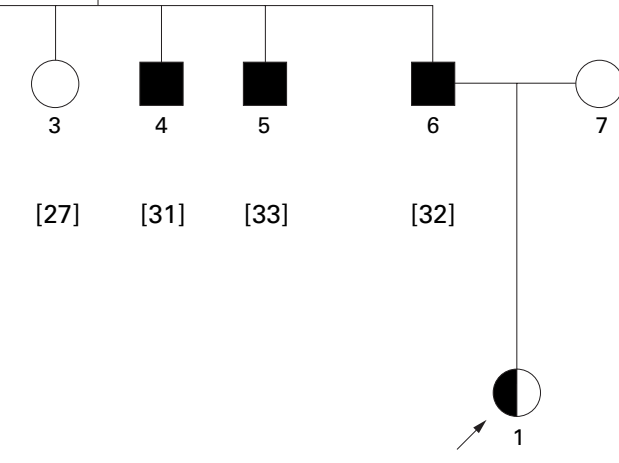

[8] tendon reflexes were absent at both ankles, and diminished at her knees and upper limbs. Plantar responses were extensor. She was ataxic on tandem gait and was unable to hop or heel walk. She had mild intention tremor of her hands. There was no pastpointing. Proprioception was abnormal in both feet but pain, temperature, and soft touch sensation were normal. She had mild dysarthria but no nystagmus and cranial nerve examination was otherwise normal. Educational psychology assessment found her to be of average ability.

The following investigations were normal: full blood picture and blood film, serum electrolytes, urea, creatinine, calcium, phosphate, alkaline phosphatase, creatinine kinase, copper and vitamin $\mathrm{E}$, lactate, immunoglobulins, $\alpha$ fetoprotein, $\beta$ lipoprotein levels, urinary and plasma amino acids, urinary organic acids, electrocardiograph, echocardiography, plain $x$ rays of chest and spine, magnetic resonance imaging of brain, and ocular slit lamp examination. Nerve conduction studies showed normal peripheral motor nerve conduction but a bilateral delay in sensory evoked responses. Chromosomal analysis confirmed a normal female karyotype $46 \mathrm{XX}$. DNA analysis was negative for the autosomal dominant spinocerebellar ataxia genes of types 1,2, 3, and 6. DNA analysis of the FRATAXIN gene in the patient showed that the GAA expansion was present in one allele but not the other, while her mother was negative for the GAA expansion in both alleles. A point mutation is currently being sought in the patient. At this stage, as the clinical diagnosis of FA was confirmed, the patient's father admitted to progressive difficulties with balance from the age of 23. DNA analysis showed he was homozygous for the FA repeat expansion.

\section{Discussion}

Genetic studies have consistently suggested that FA is inherited in an autosomal recessive manner. One study of 101 relatives of patients with FA failed to detect any neurological abnormalities in the heterozygous state. ${ }^{1}$

Much has been learnt about FA in recent years. The underlying defect is a GAA trinucleotide repeat expansion in the $\mathrm{X} 25$ gene on chromosome 9 with $90 \%$ of those studied being homozygous for expanded alleles and over half of the remaining patients being compound heterozygotes, carrying one allele with a repeat expansion and the other with a point mutation. ${ }^{2}$ Point mutations, predicted to be null alleles, are thought to be rare. ${ }^{3}$ Expanded alleles are thought to result in low or absent frataxin levels while point mutations cause single amino acid changes in the $\mathrm{C}$ terminal 
Table 1 Signs, symptoms, and investigations in family members

\begin{tabular}{|c|c|c|c|}
\hline & Father II. 6 & Paternal uncle II. 4 & Paternal uncle II. 5 \\
\hline Age at present $(y)$ & 32 & 31 & 33 \\
\hline Age at onset (y) & 23 & "Teens" & 16 \\
\hline First symptoms/signs & Backache, ataxia & Backache, ataxia & Ataxia, kyphoscoliosis \\
\hline \multicolumn{4}{|c|}{ Details of last neurological examination } \\
\hline Age & 31 & 23 & 23 \\
\hline \multicolumn{4}{|l|}{ Lower limbs } \\
\hline Tone & $\uparrow \uparrow$ & $\uparrow \uparrow$ & $\uparrow \uparrow$ \\
\hline Power & $\downarrow \downarrow$ & $\downarrow \downarrow$ & $\downarrow \downarrow$ \\
\hline Reflexes & All absent & All absent & All absent \\
\hline Plantar reflexes & $\uparrow \uparrow$ & ?? & $\uparrow \uparrow$ \\
\hline Nystagmus & Yes & Yes & None aged 23 \\
\hline Ataxic gait & Yes & Yes & Yes \\
\hline Finger nose ataxia & Yes & Yes & Yes \\
\hline Pes cavus & ++ & ++ & ++ \\
\hline Progression & Still ambulant & Ambulant at 23 & Wheelchair at 28 \\
\hline Echocardiography & $\begin{array}{l}\text { Probable early } \\
\text { cardiomyopathy }\end{array}$ & Normal at 23 & Normal at 25 \\
\hline Clinical diagnosis & FA & FA & FA \\
\hline DNA confirmation & Yes & Not done & Not done \\
\hline
\end{tabular}

FA, Friedreich's ataxia. gote for the FA gene and has developed a Friedreich's phenotype with earlier onset at 2 years. Having excluded all differential diagnoses we have to assume that she has inherited a point mutation in the X25 gene from her mother which has resulted in a form of pseudodominant inheritance. ${ }^{6}$

We support the suggestion that FA may occur in those who are heterozygous carriers for the frataxin expansion and that a diagnosis of FA should always be considered when progressive ataxic symptoms are present.

1 Harding AE. Freidreich's ataxia: a clinical and genetic study of 90 families with an analysis of early diagnostic criteria and intrafamilial clustering of clinical features. Brain 1981;104:589-620.

2 Campuzano V, Montermini L, Molto MD, et al. Friedreich's ataxia: autosomal recessive disease caused by an intronic GAA triplet repeat expansion. Science 1996;271:1423-7.

3 Monros E, Molto MD, Martinez F, et al. Phenotypic correlation and intergenerational dynamics of the Friedreich's ataxia GAA trinucleotide repeat. Am f Hum Genet 1997;61:101-10.

region of the frataxin protein, possibly resulting in a loss of function. ${ }^{4}$ Recent work suggests that frataxin is a mitochondrial protein which may have a primary or secondary effect on iron metabolism. ${ }^{45}$ These observations may offer explanations for cases described as atypical in the past. Our patient is an obligate heterozy-
Gray JV, Johnson

1997;16:323-5.
5 Babcock M, de Silva D, Oaks R, et al. Regulation of mitochondrial iron accumulation by Yfh $1 \mathrm{p}$, a putative homolog of frataxin. Science 1997;276:1709-12.

6 Harding AE, Zilkha KJ. "Pseudo-dominant" inheritance in Friedreich's ataxia. f Med Genet 1981;18:285-7.
Gray JV, Johnson KJ. Waiting for frataxin. Nat Genet

\section{Recurrent pneumonia}

Children with recurrent pneumonia usually have an underlying cause for it. A report from the Hospital for Sick Children in Toronto (Abdullah F Owayed et al. Archives of Pediatrics and Adolescent Medicine $2000 ; 154: 190-4)$ is said to include the largest series of recurrent pneumonia in children so far described.

A retrospective study of children admitted to the hospital between 1987-97 included 2952 with pneumonia, of whom 238 (8\%) had recurrent pneumonia (at least two episodes within a year or three over any period of time). An underlying cause was found in $220(92 \%)$ of these children. These causes had been known before the child developed pneumonia in $178(81 \%)$ cases, was diagnosed at the time of the first episode of pneumonia in $25(11 \%)$, and during or after a recurrence in $17(8 \%)$. The most common underlying factor was oropharyngeal incoordination and aspiration (114 children (48\%)). Almost half of these children had cerebral palsy. Their mean age at diagnosis of recurrent pneumonia was 6.3 years and all had pneumonia affecting more than one lung lobe. Other underlying problems were immune disorder (34), congenital heart disease (22), asthma (19), respiratory tract anomalies (18), and gastro-oesophageal reflux (13). No underlying cause was found in 18 children. Thirteen of the children with abnormal immune function had malignant disease, 10 had sickle cell disease, and five had HIV infection. Of the 17 children whose underlying problem was only diagnosed after recurrent pneumonia, seven had asthma, four had oropharyngeal incoordination and aspiration, three had gastro-oesophageal reflux, two had airway anomalies, and one had an immune disorder. (Children with cystic fibrosis were excluded from the series.)

Children with recurrent pneumonia should be assessed with these findings in mind. Possible underlying causes will be suggested by clinical findings and the age of the child. In this series, bilateral interstitial pneumonia on $x$ ray was suggestive of HIV infection. 